\title{
Braquimetatarsia: Revisión de la literatura a propósito de dos casos. Informe de casos
}

\author{
Brachymetatarsia: Review of the literature \\ and report of two cases. Case Report
}

\author{
Diego F. Rincón C. ${ }^{1}$, Ricardo Guzmán V. ${ }^{1}$, Lucía C. Padilla R. ${ }^{1}$, Paula A. Rincón C. ${ }^{2}$
}

Forma de citar: Rincón DF, Guzmán R, Padilla LC, Rincón PA. Braquimetatarsia: Revisión de la literatura a propósito de dos casos. Informe de casos. Rev Univ Ind Santander Salud. 2015; 47(3): 361-366. DOI: http://dx.doi.org/10.18273/revsal.v47n3-2015009 @(c) (1)

\section{RESUMEN}

La braquimetatarsia es una enfermedad en el pie, considerada rara, definida como el acortamiento anormal del metatarsiano. Su incidencia en la población está en el rango de 0.02- 0.05\%, y predomina en el sexo femenino (25:1). Existen 3 tipos: el idiopático, siendo el más frecuente; el asociado a alteraciones congénitas y el adquirido. Se describen en la literatura diversas formas de manejo, entre estos tratamientos se han trabajado el alargamiento en un tiempo y el alargamiento progresivo por medio de fijador externo. Para la selección del procedimiento adecuado para el manejo y tratamiento adecuado para los pacientes con esta sintomatología, es necesario tener en cuenta las ventajas y desventajas de cada una de ellas. En el siguiente artículo se presenta una corta revisión sobre la enfermedad y sus tratamientos, y se comentan 2 casos exitosos de alargamiento progresivo por medio de fijador externo.

Palabras Clave: braquimetatarsia, fijación externa, osteotomía, pie, metatarso.

\begin{abstract}
Brachymetatarsia is a rare foot disease, defined as the abnormal shortening of the metatarsal bone. It has a $0.02-0.05 \%$ incidence and is predominantly in females, with a $25: 1$ ratio compared to males. There are 3 types: congenital, idiopathic and associated with acquired disorders, being congenital the most common type. The current surgical options available are progressive osseous lengthening with external fixator and osteotomies with acute lengthening, each of them have advantages and disadvantages that should be taken into consideration when choosing one over the other. The following article is a short review of the illness and the report of two cases successfully treated with progressive osseous lengthening with external fixation.
\end{abstract}

Keywords: brachymetatarsia, treatment, external fixation, osteotom

1. Universidad Industrial de Santander. Bucaramanga, Colombia

2. Universidad Autónoma de Bucaramanga. Santander, Colombia

Correspondencia: Diego Fernando Rincón Cardozo. Dirección: Cra 33 \# 28 - |126 Piso 7 ortopedia. Correo electrónico: diego_ frc@hotmail.com. Teléfono: +57 3006934813 


\section{INTRODUCCIÓN}

La braquimetatarsia es una enfermedad rara en el pie $^{1-11}$, definida como el acortamiento anormal del metatarsiano $^{2}$. En 1983 Skirving y Newman reportaran la elongación de un metatarsiano con el mecanismo de fijación externa, siendo estos los primeros en exponer el uso de este método 5 . Su incidencia está en el rango $0.02 \%-0.05 \%$ y en una relación $25: 1$ en el sexo femenino con respecto a la población masculina ${ }^{3,4,8,9}$. No presenta una etiología establecida; se plantea la hipótesis de que es consecuencia de un cierre prematuro de la placa epifisiaria, llevando como consecuencia al retardo de crecimiento ${ }^{2-5,7}$. La mayoría de los casos presenta síntomas inherentes a la alteración mecánica producida por la variación del arco a nivel de las cabezas de los metatarsianos llevando a dolor y producción de callosidad. Sin embargo, la sintomatología del pie no es la principal razón de consulta; un factor importante es el efecto estético que produce el acortamiento del artejo afectado ${ }^{1,2,6,9,12}$. Existen 3 tipos: idiopático; congénito y adquirido; el más frecuente es el primero mencionado ${ }^{4}$. $\mathrm{Se}$ describen diferentes formas de manejos, de los cuales se destacan el alargamiento en un tiempo y el alargamiento progresivo por medio de fijador externos; de estos cada cual tiene ventajas y desventajas que deben tenerse en cuenta para el momento de elegir el manejo en los pacientes que presentan este tipo de patología. En este artículo referimos dos casos a quienes se realizó osteotomía metafisiaria y además alargamiento progresivo por medio de un fijador externo.

\section{PRESENTACION DE CASOS}

\section{Caso 1}

Paciente femenina de 11 años de edad sin antecedentes patológicos, traumáticos o familiares, no existe consanguinidad entre familiares. Con cuadro clínico de 7 años de evolución, evidenciado con disminución del crecimiento del cuarto artejo de pie izquierdo, acompañado de dolor durante la bipedestación y quejas estéticas ante su deformidad. Al examen físico se aprecia acortamiento de cuarto artejo sin alteración de la movilidad y dolor a la palpación. Fue llevada a osteotomía y aplicación de tutor externo (figura 1), con trasporte óseo. A los 7 días postoperatorio se inició distracción ósea con 4 cuartos de vuelta al día por 15 días, posteriormente 2 vueltas al día por 5 días y se mantuvo el fijador 11 semanas hasta evidenciar consolidación. A los 2 años, control post quirúrgico, arcos de movilidad adecuados, no dolor al deambular, cicatriz aceptable con satisfacción por parte de la paciente y los padres (figura 2) .

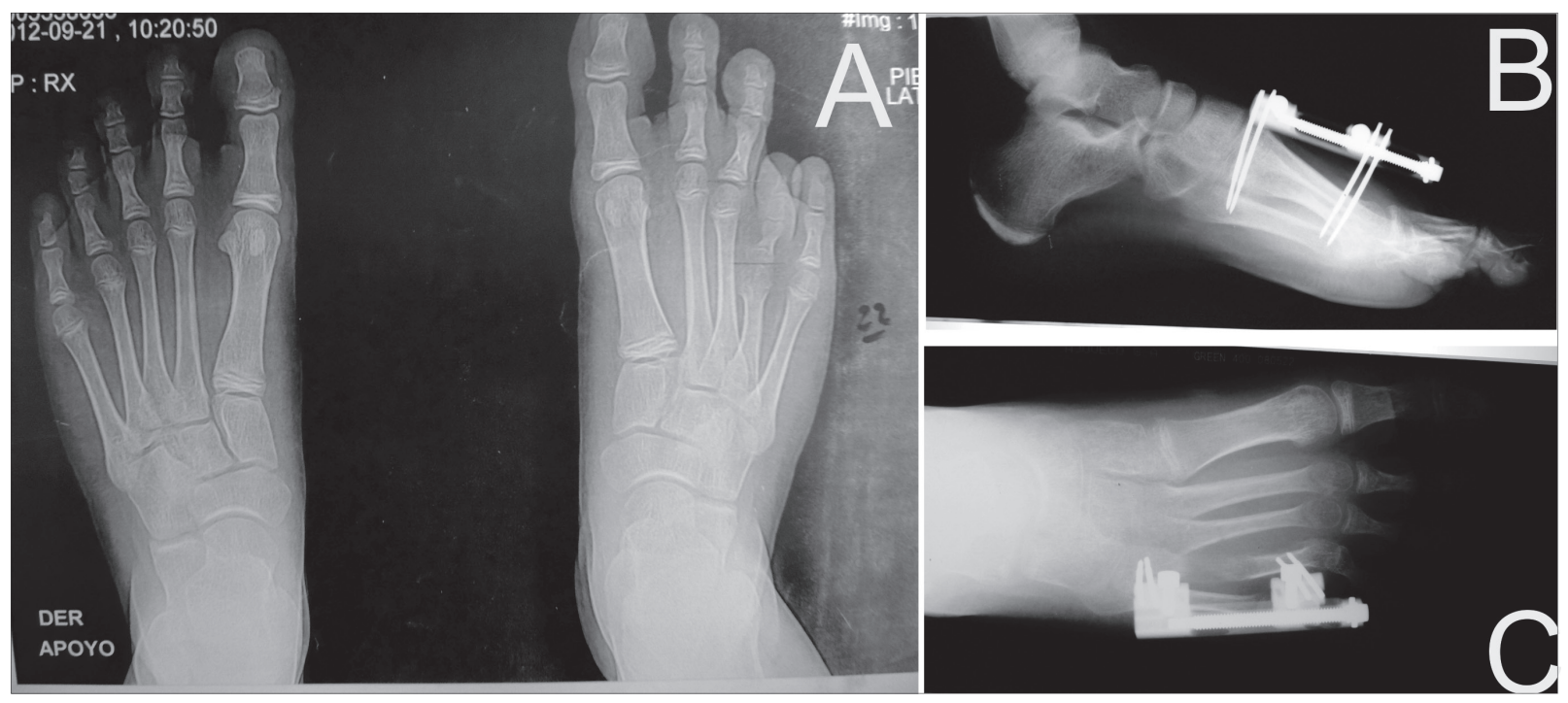

FIGURA 1. A. Rayos X Prequirúrgico: Se aprecia acortamiento del cuarto metatarsiano y alteración del arco de metatarsianos. B y C: Pie con fijador posterior a osteotomía 

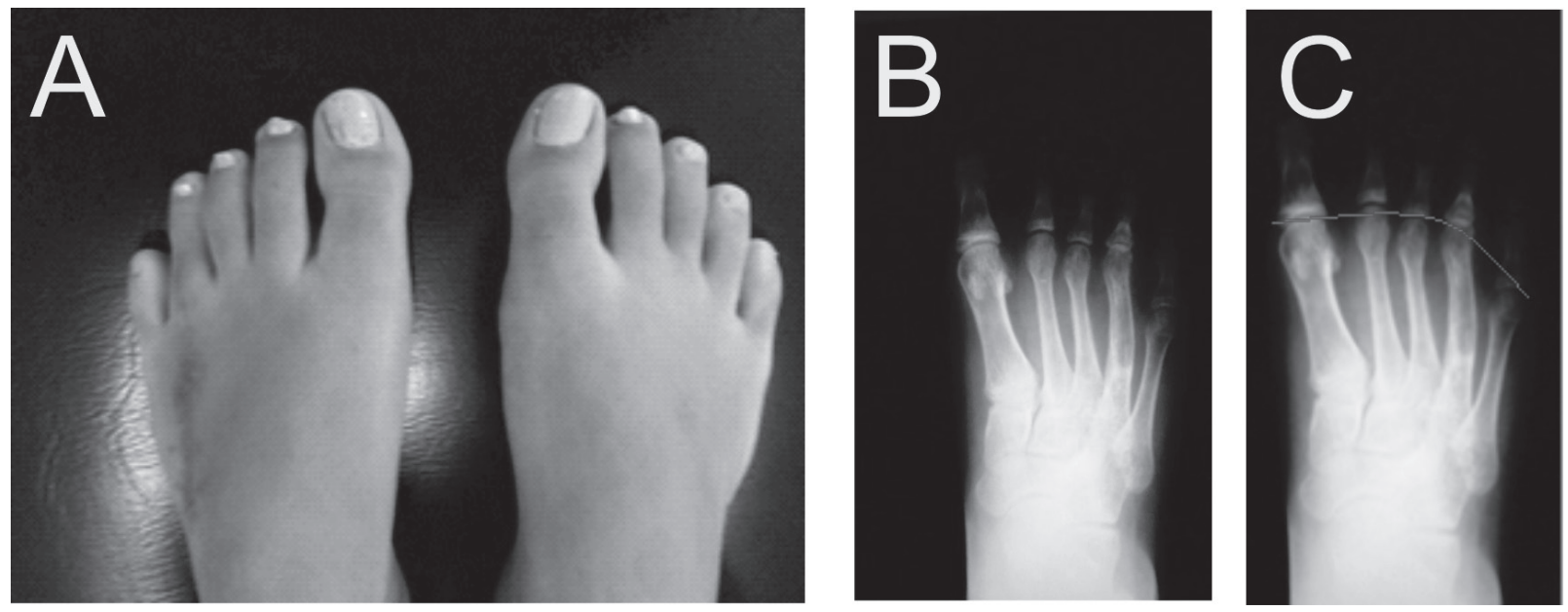

FIGURA 2. A. Pies en postoperatorio con patrones similares a ambos pies. B y C: Control post operatorio 2 años, arcos de metatarsianos congruente.

\section{Caso 2}

Paciente masculino de 12 años de edad, sin antecedentes patológicos o traumáticos, no existe consanguinidad entre familiares. Quien consulta por cuadro clínico de 8 años de evolución de crecimiento anormal del cuarto artejo pie derecho, acompañado de dolor intenso en región plantar durante actividades deportivas e incomodidad con el calzado. Fue llevado a osteotomía y aplicación de tutor externo con trasporte óseo. A los 7 días postoperatorio se inició distracción ósea con 2 cuartos de vuelta al día por 15 días; posteriormente 2 vueltas al día por 5 días. Durante la sexta semana de tratamiento presenta trauma contuso a nivel de pie con posterior aflojamiento de clavo shanz lo cual obliga a retiro prematuro. Se completa estabilización con férula posterior hasta completar 12 semanas. No se presentaron infecciones u otras complicaciones. (figura 3 )

FIGURA 3. A Radiografia postoperatoria control

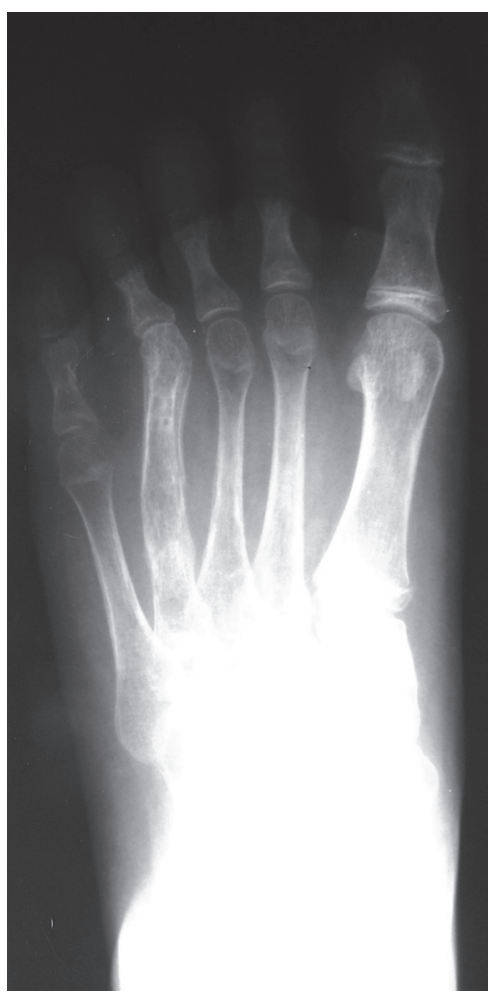




\section{DISCUSIÓN}

La braquimetatarsia es una patología poco usual; puede presentarse unilateral $o$ bilateral en un $70 . \%^{1,3,5,8,9}$. Cuando se manifiesta de manera única el metatarso más afectado es el cuarto ${ }^{4,6,7,17}$, le sigue en frecuencia el primero y el quinto ${ }^{2,9}$. Se define como el acortamiento anómalo, debe tener como característica que la epífisis distal se encuentre a 5 $\mathrm{mm}$ proximal del arco metatarsiano $0^{9,21}$ (figura 1A). Existen tres categorías para esta entidad; la primera idiopática puede asociarse con síndromes sistémicos, endocrinopatías y displasias. La braquimetatarsia adquirida es secundaria a procesos traumáticos, infección, tumores, infarto de Freiberg, lesiones por radiación o procedimientos quirúrgicos (fijación transfisiaria, osteotomía del metatarso, fijación interna o externa produciendo arresto y sinostosis entre metatarsianos, etc.); la tercera categoría asociación congénita se correlaciona con síndrome de Down, Turner, Larsen, Albright y enanismo distrófico ${ }^{2,3,4,6,7,13}$. Otros autores expresan que el proceso también puede verse asociado a hipoplasia de las falanges y del tejido en el $64.7 \%{ }^{1}$.

La presentación de la enfermedad no es usual al momento del nacimiento pero se produce la manifestación clínica a los 4 años de edad ${ }^{4}$. La mayoría de quejas del paciente es el dolor, la sensación de incomodidad con el uso de calzado, formación de callosidades dorsales, deformidad de los otros artejos para suplir el espacio perdido produciendo deformidades en varo o valgo, contracturas de tejidos adyacentes y alteraciones estéticas ${ }^{1,2,4,6,7,14,16,22}$.

La confirmación diagnóstica se identifica con radiografías del pie para determinar la pérdida del arco parabólico de metatarsiano, teniendo en cuenta el acortamiento proximal a la parábola de mínimo $5 \mathrm{~mm}^{9}$ (figura 1A).

Entre los diagnósticos diferenciales que se deben tener en cuenta antes de decidir un tratamiento definitivo son pocos, teniendo como característica la disminución del crecimiento de metatarsianos como: Displasia epifisiaria multiple, osteocondromas y la artritis reumatoidea juvenil $26,27,28$.

El manejo de esta patología a pesar de presentarse tan temprano, se debe postergar hasta los 12 años por que el crecimiento óseo del paciente debe estar completo, además el manejo postquirúrgico es más fácil. En la literatura se describen dos tipos de tratamientos para esta entidad. El alargamiento agudo, inicialmente descrito por McGlamry y Cooper en $1969^{4,7,16}$. El principio es realizar una osteotomía al metatarsiano afectado y posteriormente interponer un injerto autólogo o aloinjerto, fijando la mayoría de veces descrita con clavos, según la literatura se han presentado buenos resultados pero con riesgo de sobre estiramiento de vasos y nervios con la consecuente necrosis por el efecto distraccional agudo. Por tal motivo recomiendan no exceder el $23 \%$ de la longitud del metatarsiano $0^{4}$. Los beneficios de este manejo son: El menor tiempo para la unión y menor cicatriz, pero las desventajas son la morbilidad en el área donante, la dificultad para reconstruir la parábola de los metatarsianos, el riesgo de colapso del injerto e inestabilidad del implante ${ }^{4,17,21}$, no se puede dar apoyo inmediato, haciendo de este un método poco utilizado y de difícil consecución de resultados satisfactorios $^{8,10,11,14}$.

El otro procedimiento utilizado es el alargamiento gradual con el uso de fijador externo, actualmente es el más popular, se reportan múltiples ventajas para mejorar deformidades a nivel de las extremidades. Las metas que se desean conseguir son: alivio del dolor, pie estéticamente adecuado y restituir la función de la parábola metatarsiana ${ }^{8,13,17}$.

Los que abogan la distracción, exponen que tienen múltiples ventajas ${ }^{12}$. El alargamiento simultaneo de tejido blando y óseo, atribuyendo a este proceso la disminución del daño neurovascular; se logra la longitud deseada del metatarsiano y se restablece el arco; no requiere de injerto óseo, y la posibilidad de apoyo es inmediato ${ }^{3,4,8,14,18-20}$. Las desventajas de este procedimiento es que el proceso distraccional produce mayor tiempo para la consolidación lo que puede llevar a la mala unión o no unión, a su vez infección del trayecto de los schanz, posibilidad de fusión prematura, subluxación o luxación de la articulación metatarso falángica, angulación del metatarso y rigidez articular ${ }^{3,12,15,20}$ y se aconseja que la distracción no debe exceder el $40 \%$ de la longitud total del metatarsiano para disminuir el riesgo de luxación; si por el contrario requiere de mayor longitud sugieren el alargamiento del tendón extensor con Z plastia, el uso de un clavo de kirshner transarticular metatarso falángico para protección de luxación y si se produce rigidez la liberación plantar capsular ${ }^{1,12}$. El uso de fijadores externos también requiere de mayor tiempo de tratamiento; los familiares deben ser entrenados y el fijador puede producir poca adherencia por la dificultad de uso de calzado. 
Como consejos a la técnica se sugiere realizar incisión pequeña sin alterar de manera desordenada el periostio, realizar osteotomía metafisiaria, iniciar el alargamiento a los 7 días, $0.25 \mathrm{~mm}$ cuatro veces al día para alargar $1 \mathrm{~mm}$ diario hasta alcanzar la altura deseada y posteriormente continuar con el fijador estático por 4 a 6 semanas ${ }^{13,17-19,23}$. Recomendación de la literatura es una elongación no mayor al $40 \%$. Esto ayuda a preservar de manera máxima la vascularización perióstica y endóstica, distracción pequeña en pasos frecuentes, uso fisiológico normal de la región elongada, preservar el coagulo de osteotomía y así la migración de células osteoprogenitoras $^{13,14}$.

Entre los artículos estudiados durante esta revisión, no se encontraron otros casos documentados a nivel nacional, pero a nivel latinoamericano si encontramos revisiones de tema y casos reportados donde fueron manejados con osteotomía y fijación externa, con resultados satisfactorios. Crecimiento promedio 12-21 $\mathrm{mm}$, y el tiempo de consolidación con un rango entre 12 y 17 semanas $^{27,28}$. Comparando nuestros casos, con los reportados en la publicaciones latinoamericanas, se encontraron descritos complicaciones mininas, como: La pseudoartrosis, luxación metatarsofalangica, y metatarsalgia por alargamiento excesivo ${ }^{26,27,28}$.

La mayoría de autores reportan buenos resultados con la utilización de la osteogenesis distraccional. Se debe tener como principio de tratamiento en pacientes que requieren alargamientos de más de $1 \mathrm{~cm}^{20}$. Cabe resaltar que cada paciente debe ser tomado individualmente y dependiendo del apoyo familiar y las exigencias de cada uno para poder optar por un tipo de tratamiento ${ }^{6}$.

En los casos reportados los pacientes tuvieron satisfacción, resultados adecuados de marcha y mejoría de dolor, además de complacencia de la parte estética. En la literatura debido a que la patología no es frecuente en su mayoría son estudios de reportes de casos, sin un control funcional por escalas adecuado, por lo que no hay estudios con suficiente valor epidemiológico. El uso de fijador externo es un método fácil, práctico con buenos resultados eficaces y estéticos.

\section{REFERENCIAS}

1. Shim JS, Park SJ. Treatment of brachymetatarsia by distraction osteogenesis. J Pediatr Orthop. 2006; 26(2): 250-254.

2. Lee KB, Park HW, Chung JY, Moon ES, Jung ST, Seon JK. Comparison of the outcomes of distraction osteogenesis for first and fourth brachymetatarsia. J
Bone Joint Surg Am. 2010; 92: 2709-2718.

3. Scher DM, Blyakher A, Krantzow M. A Modified surgical technique for lengthening of a metatarsal using an external fixator. HSSJ. 2010; 6(2): 235239.

4. Giannini S, Faldini C, Pagkrati S, Miscione MT, Luciani D. One-stage metatarsal lengthening by allograft interposition: a novel approach for congenital Brachymetatarsia. Clin Orthop Relat Res. 2010; 468: 1933-1942.

5. Oribio Gallegos JA, Soto Bañuelos EA. Osteotomía de alargamiento en la braquimetatarsia. Acta Ortop Mex. 2002; 16(6): 318-320.

6. González Macías FJ. Braquimetatarsia doble bilateral, presentación de un caso tratado en forma simultánea mediante elongación ósea progresiva usando minifijadores externos. Acta Ortop Mex. 2004; 18(5): 221-223.

7. Lamm BM. Percutaneous distraction osteogenesis for treatment of brachymetatarsia. J Foot Ankle Surg. 2010; 49(2): 197-204.

8. Guizar-Cuevas S, Mora-Rios FG, Mejía-Rohenes LC. Elongation with callotaxis for congenital brachmetatarsia. Acta Ortop Mex. 2010; 24(6): 394398.

9. Choi IH, Chung MS, Baek GH, Cho TJ, Chung CY. Metatarsal lengthening in congenital brachymetatarsia: one-Stage lengthening versus lengthening by callotasis. J Pediatr Orthop. 1999; 19(5): 660-664.

10. Giannini S, Faldini C, Pagkrati S, Miscione MT, Luciani D. One-stage metatarsal lengthening by allograft interposition: a novel approach for congenital brachymetatarsia. Clin Orthop Relat Res. 2010; 468(7): 1933-1942.

11. Masada K, Fujita S, Fuji T, Ohno H. Complications following metatarsal lengthening by callus distraction for brachymetatarsia. J Pediatr Orthop. 1999; 19(3): 394-397.

12. Fox IM. Treatment of brachymetatarsia by the callus distraction method. J Foot Ankle Surg. 1998; 37(5): 391-395.

13. Desai A, Lidder S, Armitage AR, Rajaratnam SS, Skyrme AD. Brachymetatarsia of the fourth metatarsal, lengthening scarf osteotomy with bone graft. Orthop Rev. 2013; 5(3): e21.

14. Martin DE, Stran DC, Southerland JT, Wesselowski BE. Callus distraction in reconstructive foot surgery. J Foot Ankle Surg. 1996; 35(5): 489-506.

15. Brown MJ, Yeoman TF, Roberts S, Pillai A. Case report: a modified 1-stage technique for the treatment of brachymetatarsia. Foot Ankle Spec. 2012; 5(6): 389-393. 
16. Oh CW, Sharma R, Song HR, Koo KH, Kyung HS, Park BC. Complications of distraction osteogenesis in short fourth metatarsals. J Pediatr Orthop. 2003; 23(4): 484-487.

17. Houshian S, Skov O, Weeth RE. Correction of congenital brachymetatarsia by gradual callus distraction. Scand J Plast Reconstr Surg Hand Surg. 2002; 36(6): 373-375.

18. Lee KB, Yang HK, Chung JY, Moon ES, Jung ST. How to avoid complications of distraction osteogenesis for first brachymetatarsia. Acta Orthop. 2009; 80(2): 220-225.

19. Lamm BM, Gourdine-Shaw MC. Problems, obstacles, and complications of metatarsal lengthening for the treatment of brachymetatarsia. Clin Podiatr Med Surg. 2010; 27(4) 561-582.

20. Kim HT, Lee SH, Yoo CI, Kang JH, Suh JT. The management of brachymetatarsia. J Bone Joint Surg Br. 2003; 85(5): 683-690.

21. Baek GH, Chung MS. The treatment of congenital brachymetatarsia by one-stage lengthening. J Bone Joint Surg Br. 1998; 80(6): 1040-1046.

22. Yamada N, Yasuda Y, Hashimoto N, Iwashiro H, Uchinuma E. Use of internal callus distraction in the treatment of congenital brachymetatarsia. Br J Plast Surg. 2005; 58(7): 1014-1019.
23. Smolle E, Scheipl S, Leithner A, Radl R. Management of congenital fourth brachymetatarsia by additive autologous lengthening osteotomy (AALO): A case series. Foot Ankle Int. 2015; 36(3): 325-329.

24. Haleem AA, Berry DJ, Hanssen AD. The Chitranjan ranawat award: mid-term to long-term followup of two-stage reimplantation for infected total knee arthroplasty. Clin Orthop Relat Res. 2004; 428: 35-39.

25. Kim HN, Jeon JY, Dong Q, Kim HK, Park YW. Prevention of cavus foot deformity following gradual distraction osteogenesis for first brachymetatarsiatechnique tip. Foot Ankle Int. 2014; 35(3): 300-303.

26. D'Arrigo A, Arenas Planelles A, Eraso Lara I, Jiménez Sarmiento O. La braquimetatarsia: presentación de un caso. Rev Españ Cirug Osteoart. 2011; 246(46): 69-72.

27. Remón Dávila XJ, Peña Marrero L, López Díaz H, Álvarez Cambras R, Rodrigo Olarán H, Rodríguez Rodríguez A. Tratamiento de la braquimetatarsia mediante elongación ósea con minifijador externo RALCA®. Rev Cubana Ortop Traumatol. 2008; 22(2): 1-8.

28. Zabalaga J. Tratamiento quirúrgico de discrepancia metatarsal. Rev Boliv Ortop Traumatol. 2007; 17(1): 56-60. 